\title{
DE LA VULNERABILIDAD DE LOS JÓVENES A NO EDUCARSE: UNA APROXIMACIÓN CUANTITATIVA PARA EL CASO COLOMBIANO
}

\author{
Por: Richard Iván López Zambrano ${ }^{1}$
}

\begin{abstract}
RESUMEN
Este artículo presenta un análisis de los determinantes de la vulnerabilidad en los jóvenes entre 14 y 28 años. Tomando como base el enfoque de desarrollo humano, se explica la vulnerabilidad desde la dimensión de educación, lo que sugiere que un joven puede ser vulnerable a no educarse ya sea por analfabetismo o por inasistencia escolar, la selección de la dimensión redunda en la importancia que tiene la misma en una sociedad como motor de desarrollo.

El estudio se realiza a través de un análisis microeconométrico haciendo uso de modelos con variable dependiente limitada. Los principales hallazgos demuestran que la proporción de analfabetismo aunque es baja, se explica por la falta de dinero, costos de educación y porque debe trabajar; además, se asocia directamente con los bajos niveles de educación del padre. Por su parte, la inasistencia escolar la padecen en mayor medida las mujeres que los hombres. Así mismo, en la medida en que el padre incrementa su nivel de educación y además el hogar está compuesto por una madre, el joven tiene una mayor probabilidad de estudiar.
\end{abstract}

Palabras clave: Vulnerabilidad, pobreza, educación, modelos binarios.

JEL: 010

1. Economista graduado con tesis meritoria de la Universidad de Nariño. Investigador, Grupo de Investigación Frontera Sur adscrito a la Facultad de Ciencias Económicas y Administrativas de la Universidad de Nariño, Clasificado en Colciencias con Categoría D. E-mail: rlopez3@udenar.edu. co. 


\title{
VULNERABILITY ASSESSMENT OF YOUNG PEOPLE NOT TO EDUCATE: A QUANTITATIVE APPROACH FOR THE COLOMBIAN CASE
}

\author{
By: Richard Iván López Zambrano
}

\begin{abstract}
This paper presents an analysis of the determinants of vulnerability among young people between 14 and 28 years. Based on the human development approach, vulnerability is explained from the perspective of education, suggesting that a young person can be vulnerable to not educated either illiteracy or truancy, selecting the dimension is in the importance It has the same engine in a society like development.

The study is conducted through a microeconometric analysis using models with limited dependent variable. The main findings show that although the proportion of illiteracy is low, can be explained by lack of money, education costs and that should work; furthermore, it is directly associated with low levels of parent education. For its part, the truancy suffer further women than men. Also, to the extent that the parent increased their level of education and also the home is composed of a mother, the young are more likely to study.
\end{abstract}

Keywords: Vulnerability, poverty, education, binary models.

JEL: 010 . 


\title{
AVALIAÇÃO DA VULNERABILIDADE DE JOVENS A NÃO SE EDUCAR: UMA APROXIMAÇÃO QUANTITATIVA PARA O CASO COLOMBIANO
}

\author{
Por: Richard Iván López Zambrano
}

\section{RESUMO}

Este trabalho apresenta uma análise dos determinantes da vulnerabilidade entre jovens entre 14 e 28 anos. Com base na abordagem do desenvolvimento humano, a vulnerabilidade é explicado a partir da perspectiva da educação, o que sugere que um jovem pode ser vulnerável a não educados ou analfabetismo ou a evasão escolar, selecionando a dimensão é na importância Ele tem o mesmo motor em uma sociedade como o desenvolvimento.

O estudo é realizado através de uma análise microeconométrica usando modelos com variável dependente limitada. As principais conclusões mostram que, embora a proporção de analfabetismo é baixo, pode ser explicado pela falta de dinheiro, os custos da educação e que deve funcionar; além disso, está diretamente associado com baixos níveis de educação dos pais. Por seu lado, a evasão escolar sofrem mais mulheres do que homens. Além disso, na medida em que o pai aumentou seu nível de educação e também a casa é composta de uma mãe, os jovens são mais propensos a estudar.

Palavras-chave: Vulnerabilidade, pobreza, educação, modelos binários.

JEL: O10. 


\section{INTRODUCCIÓN}

La situación de los jóvenes en el mundo reclama la atención de las autoridades de distintos órdenes, todos los actores son llamados a hacerle frente a las diferentes problemáticas que se evidencian en la población joven. En efecto, la Organización de las Naciones Unidas (ONU) sostiene que "los jóvenes son un factor determinante en el ámbito social, el desarrollo económico y el progreso técnico. Su imaginación, sus ideales, sus perspectivas y su energía resultan imprescindibles para el desarrollo de las sociedades en las que viven".

El planteamiento de ONU, sin duda trae implícito el componente educativo, pues no será posible el desarrollo económico y el progreso técnico si no se tiene una juventud educada con calidad; de ahí que sea insoslayable multiplicar los esfuerzos encaminados a crear y desarrollar políticas y programas destinados a los jóvenes, tal y como señala ONU, las medidas que se implementen para tratar el tema de la juventud y aprovechar al máximo su potencial, repercutirán en la situación socioeconómica actual, así como en el bienestar de las generaciones futuras.

Por su parte, la Organización Internacional del Trabajo -OIT- (2015) señala en un informe sobre Juventud e Informalidad, intitulado: "Formalizando la Informalidad Juvenil, Experiencias innovadoras en América Latina y el Caribe" que, "la informalidad en el empleo juvenil representa una preocupación doble para la agenda de los países de América Latina y el Caribe. Al desafío de brindar a la nueva fuerza laboral un lugar activo en el mercado de trabajo se agrega el de garantizar que el mismo reúna los atributos de un trabajo decente" esta premisa concuerda con lo planteado por ONU, pues precisamente, "la educación permite a los jóvenes acceder a un trabajo decente, salir de la pobreza y alcanzar un nivel de vida satisfactorio."

En ese orden, a nivel nacional se han realizado importantes esfuerzos para mejorar las condiciones de los jóvenes, relacionados con el empoderamiento, la democracia participativa y la ciudadanía activa. Para ello, el Gobierno Nacional ha venido impulsando la creación de los Consejos Municipales de Juventud (CMJ), Plataformas Juveniles, Políticas Públicas de Adolescencia y Juventud, Cabildo Joven y el Presupuesto Participativo, entre muchos otros, espacios que contribuyen a fortalecer el diseño de política pública haciendo partícipes a los jóvenes de las situaciones que les afectan.

Adicionalmente, el Gobierno Nacional, consciente de las necesidades de la población joven en el concierto económico actual, en febrero del año 2015 realizó tres importantes anuncios a través del Ministerio de Trabajo; entre ellos, se lanzó el programa 40.000 Primeros Empleos, dirigido a población entre 18 y 28 años sin experiencia laboral, recién egresados de algún programa de Educación Superior y bachilleres que no hayan podido continuar con su proceso de formación en las regiones con mayor tasa de desempleo juvenil; el programa Transporte para el Empleo, el cual permite que a partir del nueve de febrero, las personas que visiten un centro de empleo en Bogotá, Cali y Medellín y que deban visitar empresas para presentar entrevistas pero no tengan la posibilidad de pagar el transporte, reciban una tarjeta con un cupo cercano a los 24 mil pesos. Finalmente, el tercer anuncio tiene que ver con el programa Talentos para el Empleo que ofrece 13 mil cursos de formación, diferentes a los que oferta el SENA, en habilidades para el trabajo a 
personas entre 18 y 45 años. Son cursos de validación del bachillerato, manejo de computadores y finanzas, habilidades que han sido identificadas como esenciales para la búsqueda de empleo (Mintrabajo, febrero de 2015).

En el marco de las metas, objetivos y proyectos del gobierno en torno a los jóvenes, se ha creado el Estatuto de Ciudadanía Juvenil, Ley Estatutaria 1622 de 2013, la cual deroga la Ley de Juventud 375 de 1997, su aplicación aún es objeto de discusión y diálogo que en todo caso, motivan a garantizar los derechos fundamentales de la población juvenil.

Así, el presente estudio toma como referencia el concepto de joven señalado por la Ley, esto con el fin de definir el rango de edad objeto de estudio. El Artículo $5^{\circ}$ sobre definiciones, reza: "joven es toda persona entre 14 y 28 años cumplidos en su condición de su autonomía intelectual, física, moral, económica, social y cultural que hace parte de una comunidad política y en ese sentido ejerce su ciudadanía”.

En esa medida la presente investigación contribuye a comprender las problemáticas que padecen los jóvenes desde el plano de la vulnerabilidad; este tipo de contribuciones coadyuvan al diseño de políticas públicas con enfoque diferencial, pertinentes y efectivas. Se inicia identificando las causas y efectos que provoca un estado de vulnerabilidad en la población joven, en aras de superar la pobreza y evitar su perpetuidad o evitar caer en ella mediante el diseño de planes, programas y proyectos construidos desde y para los jóvenes.

Este trabajo se ha dividido en cinco partes, la primera de ellas corresponde a esta introducción; a continuación se estudia algunos referentes teóricos relevantes que sustentan el marco de estudio; luego sigue la metodología relacionada principalmente con el modelaje econométrico; enseguida se presentan los resultados y finalmente se realizan algunos comentarios finales.

\section{MARCO TEÓRICO}

La literatura respecto al tema de la vulnerabilidad aún se encuentra en un estado exploratorio, razón por la cual se observa una serie de estudios que no logran consolidar un cuerpo teórico solido; en efecto, los esfuerzos de investigadores de distintas disciplinas han centrado su atención en la pobreza como factor determinante. En esta línea, el Conpes Social 100 (2006: 10) sostiene que cuando se trata de diseñar programas y definir instrumentos, se encuentra con que no existen criterios técnicos unívocos, por lo cual los operadores locales como regionales serían quienes adapten los conceptos a la realidad de las variables.

El mismo documento (Conpes Social 100, 2006: 12), señala en cuanto a las políticas de focalización, la importancia de las definiciones e indicadores de pobreza, así mismo aduce que "los programas que tienen como objetivo lograr que un grupo poblacional supere una condición de vulnerabilidad, deben avanzar en la definición conceptual de vulnerabilidad, condiciones de vulnerabilidad y construcción e implementación de mediciones que posibiliten la focalización”, por lo cual el documento señalado termina destacando la importancia de adelantar estudios encaminados a mejorar la valoración de las condiciones de vulnerabilidad de la población. 
En ese orden, las políticas públicas se han enfocado a resolver problemas existentes; es decir, las políticas han sido más de corte curativo que preventivo. La ventaja de analizar la problemática de la pobreza desde la óptica de la vulnerabilidad, permite anticiparse a los riesgos asociados a dicho estado negativo relacionados fundamentalmente con la probabilidad y el riesgo de ser pobre e incluso de perpetuarse el fenómeno.

A pesar de los escasos estudios y la discusión que existe respecto al tema de la vulnerabilidad, situación que no dista de la presentada en el tema de la pobreza donde tampoco existe una definición estructurada y concluida, conviene en este caso revisar algunas conceptualizaciones. Al respecto, Busso (2001) señala que:

La vulnerabilidad es compleja, multicausal, tiene varias dimensiones analíticas e incluye aspectos de individuos y hogares así como también características económicas, políticas, culturales y ambientales de la sociedad. Por ello, la vulnerabilidad tiene carácter polisémico y da lugar a múltiples enfoques metodológicos constituyendo en sí misma un sistema dinámico (Citado por: Estupiñán y Guerrero, 2013: 31).

Castaño (2007), en un estudio realizado para el Departamento Nacional de Planeación (DNP), señala que la vulnerabilidad es uno de los aspectos de la pobreza, la autora advierte que el concepto tiene que ver con el nivel en el que uno o varios individuos pueden resultar afectados como consecuencia de un evento de peligro. En efecto, define la vulnerabilidad como: el "grado en el cual un hogar o individuo sufre de uno o más episodios de pobreza o la persistencia de esta, como consecuencia de un estímulo o shock. La pobreza será entendida como aquella que ocurre cuando una persona experimenta una privación fundamental, es decir, la carencia de las capacidades básicas o esenciales para su bien-estar (CPRC, p: 5).

Por su parte, un informe del Banco Mundial (2000), plantea que la vulnerabilidad mide "la capacidad de resistencia ante una crisis [y] la probabilidad de que una crisis dé por resultado una disminución del bienestar" (citado por: Castaño, 2007: 6). De manera que, si la vulnerabilidad mide la capacidad de resistencia frente a las crisis, es porque los individuos son activos y dinámicos, lo que los lleva a reaccionar frente a los peligros. Así, según la interpretación de Castaño, las formas y los factores que determinan estas respuestas son los aspectos centrales para reducir la vulnerabilidad y por consiguiente, el impacto de los eventos de peligro o los riesgos.

Siguiendo a Turner et al. (2003), el riesgo es una función de: i.) La perturbación y ii.) La vulnerabilidad de la unidad expuesta, es decir:

A) Riesgo $=f$ (perturbación, vulnerabilidad de la unidad expuesta)

B) Vulnerabilidad $=f$ (exposición al peligro, capacidad del sistema para manejar el shock) 
Reemplazando A.) en B.)

C) Riesgo $=f$ (perturbación, exposición al peligro, capacidad del sistema para manejar el shock).

En ese orden,

(...) el proceso que lleva al desastre o impacto final del evento de peligro se puede analizar en cuatro tiempos: i.) El evento de peligro y la exposición a este; ii.) La sensibilidad del sistema -la magnitud de su respuesta a este evento; iii.) El impacto inicial sobre el sistema o la población y su reacción o proceso de ajuste (resiliencia), y iv.) El impacto final luego del ajuste. En este caso la vulnerabilidad es un concepto explícito porque es la vulnerabilidad de las poblaciones, es decir capacidad de respuesta, la que en últimas genera el impacto final sobre el hogar o el individuo (Citado por: Castaño, 2007: 6).

Según Turner (2003), la vulnerabilidad no es un estado estacionario, sino que por el contrario es un concepto dinámico que implica un proceso en el tiempo, una cadena de acontecimientos. La vulnerabilidad será entendida entonces, como el grado en el cual un país, una región, un individuo, una comunidad o un hogar, en términos generales, determinada población, resultan o pueden resultar perjudicados como consecuencia de un estímulo o evento de peligro.

Heitzmann et al. (2002), (Citado por: Castaño, 2007), sostiene que el concepto señalado implica tres dimensiones bien específicas: i.) La necesidad de establecer qué determinará que un suceso sea definido como negativo; ii.) Los determinantes de la vulnerabilidad y iii.) Las formas de medición e identificación de sistemas y/o poblaciones vulnerables.

Las dimensiones citadas plantean una serie de interrogantes que motivan este aporte investigativo, entre ellas: ¿Cuál es el enfoque sobre el que se evaluarán los cambios? ¿Qué se considerará como un resultado que perjudicó al sistema o lo deterioró después del shock? ¿Qué factores determinan que un sistema resulte o pueda ser perjudicado luego de un shock? ¿Cómo identificar si un sistema es vulnerable o no y en qué grado? Es decir, ¿Cómo establecer la probabilidad de que un sistema llegue a una situación en la cual resulte perjudicado?

En ese sentido, la presente investigación pretende contribuir a aclarar estos y muchos otros interrogantes planteados, con el propósito de avanzar en el diseño de políticas públicas efectivas para prevenir y/o mitigar estados negativos presentados en los múltiples sistemas, a fin de mejorar la calidad de vida de los distintos grupos y sectores poblacionales. Así mismo, avanza en el estudio de estos fenómenos que plantean retos de medición interesantes.

\subsection{DETERMINANTES DE LA VULNERABILIDAD}

Una revisión a los determinantes de la vulnerabilidad es preciso realizarla desde el informe Beveridge, el cual aboga por la protección social en medio de la II Guerra Mundial, dentro de las políticas públicas y su diseño frente a las problemáticas presentadas, se realiza un diagnóstico que da cuenta sobre la indigencia en el 
Reino Unido, y concluye que se debía a la interrupción o pérdida de la capacidad para ganar dinero o a la gran cantidad de hijos. Hoy por hoy, la seguridad social se estructura en torno a la vulnerabilidad asociada con los riesgos laborales.

La seguridad social se definía como el mantenimiento de los ingresos necesarios para la subsistencia con el objetivo de eliminar la indigencia física y para mejorar la cantidad y la calidad de la población, nivel que no debía entenderse como estático, ya que los umbrales mínimos deberían progresar en todos los campos. Se proponían tasas del beneficio de subsistencia y de contribución uniformes, y los diferentes beneficios se establecían según las características del individuo con el empleo de la población (Beveridge, 1994: 73-75, 152 y 172) (Citado por: Sojo, 2012: 6).

Así, el mercado laboral sobresale en importancia como determinante de la vulnerabilidad, el cual sigue y seguirá siendo vigente en muchas dimensiones. Esto, porque en América Latina cerca del 80\% depende de los ingresos provenientes de su trabajo. No obstante, los sistemas, experimentan múltiples vulnerabilidades que interactúan entre sí y que pueden a veces combinarse, lo cual plantea desafíos en términos de un enfoque interdisciplinario que permita analizar estas dimensiones y su interacción (Summer and Mallet, 2011) (Citado por: Sojo, 2012: 6).

En razón a lo anterior, es posible encontrar múltiples tipos de vulnerabilidad, siguiendo a Castaño (2007), un análisis de los determinantes de la vulnerabilidad se pueden entender a la luz de la relación que existe entre pobreza y vulnerabilidad, entendida esta como la probabilidad que existe de ser pobre, o si estando pobre, la posibilidad que existe de perpetuarse en ese estado negativo.

En consecuencia, es importante comprender un primer escenario en donde se plantea el riesgo o la probabilidad que existe que un sistema sea pobre, de esa manera la vulnerabilidad no solo debe entenderse como una dimensión de la pobreza, sino más bien como uno de sus síntomas y al mismo tiempo una de sus causas. Esto sucede porque la existencia de riesgos y de shocks tiene importantes implicaciones en las condiciones de vida de las personas.

Partiendo de la racionalidad de los agentes, las causas de la pobreza en el marco de la vulnerabilidad, se observan cuando se analizan las acciones que toman los sistemas para reducir el riesgo y mitigar su impacto en caso de que éste efectivamente se convierta en un shock. Entre las decisiones de los agentes para prevenir el riesgo se encuentran la de diversificar sus medios de vida (v. gr. buscar varias fuentes de ingresos); dedicarse a actividades de bajos niveles de riesgo pero menos productivas, o propiciar la migración de algunos de sus miembros, entre otros (Dercon 2005; Mosser 1998) (Citado por Castaño, 2007: 8).

De otra parte, es interesante ver como los hogares actúan frente a los riesgos como la pérdida de activos por causa de un shock negativo. Los hogares ante situaciones de crisis, venden sus activos y/o sufren pérdidas de capital humano (v. gr. sacar a los niños del colegio) para dar respuesta a la situación y reducir la carga de gastos. Sin duda esta situación tiene implicaciones inmediatas y futuras sobre la capacidad de los hogares para sobrevivir y/o mantener un mejor nivel de bienestar. De esta manera la vulnerabilidad es causa importante de la persistencia y de las trampas de la pobreza. Es decir, los hogares pueden sufrir efectos permanentes de eventos transitorios (Dercon, 2005) (Citado por: Castaño, 2007: 9). 
Respecto a los determinantes de la vulnerabilidad, se puede también partir del concepto de pobreza entendido de forma multidimensional. En ese sentido, la presente aplicación aunque no realiza todo un despliegue de la propuesta de Castaño, si se convierte en el referente principal; de esta manera, se considera una serie de dimensiones básicas fundamentales asociadas al marco del Indicador de Desarrollo Humano (IDH), como son: educación, salud, y riqueza material. Cabe aclarar que en el estudio sobre una Aproximación de la Vulnerabilidad en Colombia de Castaño la riqueza material se entiende como la oportunidad de tener medios de sustento para que los individuos lleven la vida que valoran, esto es, las oportunidades de tener un empleo o una ocupación productiva.

Partiendo de lo anterior, para efectos de este trabajo se tendrá en cuenta la dimensión de educación por considerarse factor fundamental del desarrollo de una sociedad. No obstante, resulta importante revisar las condiciones que se enmarcan en cada una de las dimensiones antes citadas (ver Cuadro 1).

En este análisis se circunscribe la dimensión de educación, aspecto principal en el mejoramiento de las condiciones de vida, y aunque la dimensión salud y las condiciones adecuadas de sustento son consustanciales a la vulnerabilidad, su análisis se excluye dado el propósito del trabajo, a más de que los indicadores propuestos, en el caso de la dimensión salud, distan de los alcances de la Gran Encuesta Integrada de Hogares (GEIH) y de la Encuesta Nacional de Calidad de Vida (ENCV). No obstante, en vista de que no existe un marco teórico comúnmente aceptado, en este aspecto, como ocurre con muchos otros conceptos, es viable dejarlo a criterio del investigador, la definición de los indicadores pertinentes.

\section{METODOLOGÍA}

El presente estudio acude fundamentalmente a métodos estadísticos inferenciales, los cuales teniendo en cuenta que la información es en esencia de corte transversal, y partiendo del objetivo de analizar los determinantes de la vulnerabilidad de no tener educación, se emplea métodos econométricos caracterizados porque la variable que se desea explicar es de naturaleza cualitativa; para ello se acude a los modelos de elección discreta, modelos de elección binaria o modelos con variable dependiente limitada.

Antes de realizar una breve descripción de los modelos a utilizar, es importante señalar la razón de su utilización, se los emplea porque la variable que se desea explicar es de naturaleza dicótoma, y aunque matemáticamente se puede acudir al Modelo Clásico de Regresión Lineal (MCRL), resulta inapropiado por cuanto la estimación de este tipo de modelos por Mínimos Cuadrados Ordinarios (MCO), según Gujarati y Porter (2010), presentan los siguientes problemas: a.) No normalidad de las perturbaciones; b.) Varianzas heteroscedásticas de las perturbaciones; c.) No cumplimiento de $0 \leq E\left(Y_{i} \mid X\right) \leq 1$; esto simplemente significa que dado que los modelos lineales de probabilidad, miden la probabilidad condicional de que ocurra el evento Y dado X, éste debe encontrarse necesariamente entre 0 y 1 , por lo cual todas las probabilidades que se generen deberán cumplir con tal condición. Y finalmente, d) El coeficiente de determinación $\left(\mathrm{R}^{2}\right)$ no es una medida de bondad de ajuste confiable para modelos cuya dependiente es binaria. 
Cuadro 1. Condiciones de que un sistema sea vulnerable por no tener buena salud, no educarse y no tener adecuados medios de sustento.

\begin{tabular}{|c|c|c|}
\hline Dimensión & Condiciones & Características \\
\hline \multirow{3}{*}{$\begin{array}{l}\text { Vulnerabilidad a } \\
\text { no tener buena } \\
\text { salud. }\end{array}$} & $\begin{array}{l}\text { Enfermedades transmisibles } \\
\text { y no transmisibles. }\end{array}$ & $\begin{array}{l}\text { Sufre una enfermedad transmisible. } \\
\text { Sufre una enfermedad no transmi- } \\
\text { sible. }\end{array}$ \\
\hline & Lesiones & Es víctima de una lesión. \\
\hline & Limitaciones & $\begin{array}{l}\text { Tiene una limitación auditiva, visual } \\
\text { o sicomotora. }\end{array}$ \\
\hline \multirow{6}{*}{$\begin{array}{l}\text { Vulnerabilidad a } \\
\text { no educarse. }\end{array}$} & Educación inicial & $\begin{array}{l}\text { Es un niño mayor de } 3 \text { años y menor } \\
\text { de } 6 \text { y no asiste a un establecimiento } \\
\text { de educación inicial. }\end{array}$ \\
\hline & \multirow{2}{*}{ Educación básica y media } & $\begin{array}{l}\text { Es un niño mayor de } 7 \text { años y menor } \\
\text { de } 11 \text { y no cursa la educación básica } \\
\text { primaria. }\end{array}$ \\
\hline & & $\begin{array}{l}\text { Es un joven mayor de } 12 \text { años y me- } \\
\text { nor de } 17 \text { y no cursa educación bási- } \\
\text { ca secundaria o media. }\end{array}$ \\
\hline & Educación superior & $\begin{array}{l}\text { Es un individuo mayor de } 18 \text { años y } \\
\text { menor de } 24 \text { y no cursa alguna mo- } \\
\text { dalidad de educación superior. }\end{array}$ \\
\hline & Asistencia en general & $\begin{array}{l}\text { Es una persona que se encuentra en } \\
\text { edad escolar y no asiste a ninguna } \\
\text { institución educativa. }\end{array}$ \\
\hline & Calidad & $\begin{array}{l}\text { Es un individuo que asiste a una ins- } \\
\text { titución educativa pero no recibe } \\
\text { una educación de calidad. }\end{array}$ \\
\hline \multirow{3}{*}{$\begin{array}{l}\text { Vulnerabilidad a } \\
\text { no tener adecua- } \\
\text { dos medios de } \\
\text { sustento. }\end{array}$} & \multirow{2}{*}{ Actividad productiva } & Se encuentra desempleado. \\
\hline & & Se encuentra subempleado. \\
\hline & Nivel de ingresos & $\begin{array}{l}\text { Sus ingresos están por debajo de la } \\
\text { línea de pobreza. }\end{array}$ \\
\hline
\end{tabular}

Fuente: Castaño (2007) Departamento Nacional de Planeación, Una aproximación a la vulnerabilidad.

En razón a lo anterior, se acude a los modelos no lineales con variable dependiente limitada Logit y Probit, los cuales a diferencia de la estimación por mínimos cuadrados ordinarios que se da en el modelo clásico de regresión lineal, estos se estiman por el método de Máxima Verosimilitud², "el objetivo principal de cada uno es encontrar la probabilidad de que un acontecimiento suceda dado un conjunto de variables explicativas” (Rosales, et al. 2013: 107).

2. Explicar la metodología de este método desborda los propósitos de esta investigación. Para ampliar esta información revisar: Introducción a la Econometría. Un enfoque moderno. $4^{\mathrm{a}}$. Edición. Cengage Learning, pp. 890. Wooldridge (2010: 578). 
La ventaja de estimar a través de modelos Logit o Probit es que permiten, después de realizar algunas transformaciones ${ }^{3}$ a los valores estimados, analizar en términos de probabilidades, esto resulta muy útil para el propósito planteado en esta investigación, en la medida en que la metodología propuesta, aporta a comprender el fenómeno de la vulnerabilidad en Colombia, a partir del análisis de sus determinantes microeconómicos, esto admite v. gr., establecer la probabilidad que existe de ser pobre o de seguir siendo pobre.

Según la literatura, no existen criterios claros para seleccionar un modelo logit o probit, teniendo en cuenta que la diferencia entre los estimadores es realmente mínima, muchos investigadores optan por correr los dos modelos y posteriormente evalúan el modelo con mejor ajuste a partir de indicadores como la Razón de Verosimilitud (RV); la capacidad predictiva del modelo y el Pseudo R².

No obstante, para el caso del presente trabajo se ha seleccionado un modelo probit, las razones obedecen, aparte de lo expuesto, a que la distribución que sigue este modelo es una normal, lo que da al investigador una mayor confianza al interpretar factores acumulados que no se pueden medir, mientras que un modelo logit, que a propósito sigue una distribución logística, se asemeja a la normal, pero no lo es.

Para efectos de análisis, se emplea datos anonimizados pertenecientes al DANE que provienen de las encuestas: Gran Encuesta Integrada de Hogares (GEIH) y la Encuesta Nacional de Calidad de Vida (ENCV) ${ }^{4}$. Dado que la población objeto de estudio de la presente investigación son los jóvenes entre 14 y 28 años de edad, se procedió a filtrar la base de datos, obteniendo así un total de 18.777 observaciones para el año 2012.

\subsection{DIMENSIONES}

De acuerdo al criterio del autor del estudio, el análisis se refiere a la dimensión de vulnerabilidad a no educarse, los indicadores propuestos se pueden observar en el cuadro 2. Siguiendo a Castaño (2007), el objetivo de política en este punto es que las personas reciban una educación de calidad de acuerdo con sus condiciones y su edad; el análisis de los determinantes de los fenómenos planteados resulta de gran importancia en la medida en que contribuyen al diseño de política pública.

\section{Cuadro 2. Indicadores de educación}

\begin{tabular}{|l|l|l|c|}
\hline \multicolumn{1}{|c|}{ Objetivo } & \multicolumn{1}{|c|}{ Indicador propuesto } & Fuente & Año \\
\hline $\begin{array}{l}\text { Evaluar el acceso a una } \\
\text { educación de calidad. }\end{array}$ & Analfabetismo (Mayores de 14 años) & (GEIH) y (ENCV) & 2012 \\
\cline { 2 - 4 } & Inasistencia escolar & (GEIH) y (ENCV) & 2012 \\
\hline
\end{tabular}

Fuente: el autor, a partir del estudio de Castaño (2007) DNP.

3. Se realiza a través de las derivadas de la probabilidad del modelo probit, las cuales reflejan los cambios en la probabilidad de que $Y=1$ que ocurren cuando cambia cada una de las variables independientes $X_{k}$. Es decir, la derivada de la probabilidad de $Y$ respecto a $X_{k}$ es igual a: $(\partial \operatorname{Pr}(Y i=$

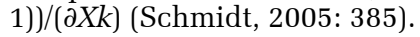

4. El empalme de las encuestas fueron realizadas por el docente investigador del Centro de Desarrollo Regional y Empresarial -CEDRE- de la Universidad de Nariño. Ms. En Economía del Desarrollo, Víctor Jaramillo Mejía. 


\subsection{ESPECIFICACIÓN DEL MODELO ECONOMÉTRICO}

Con el propósito de explicar el fenómeno objeto de estudio, se emplea características de los individuos y hogares, así, el análisis en consecuencia es de naturaleza microeconométrica. La especificación general del modelo se determina en la ecuación (a).

$$
Y_{i}=D_{i} \beta+W_{i} \beta+\mu_{i}(\boldsymbol{a})
$$

En donde, $\boldsymbol{Y}_{i}$ es una variable dependiente cualitativa que toma el valor de 1 si el individuo es analfabeto (no sabe leer o escribir) y 0 en caso contrario; $\boldsymbol{D}_{\boldsymbol{i}}$ representa el conjunto de variables explicativas de naturaleza dicótoma; $\boldsymbol{W}_{\boldsymbol{i}}$ constituye una variable ordinal, en este caso, la edad; $\mu_{i}$ es el termino de perturbación, el cual recoge la incidencia de las variables que fueron omitidas en el modelo. La especificación de las variables se puede observar en el cuadro 3.

\section{Cuadro 3. Especificación de variables del modelo de Vulnerabilidad a no educarse por analfabetismo.}

\begin{tabular}{|c|c|c|}
\hline $\begin{array}{c}\text { Variable en } \\
\text { el modelo }\end{array}$ & Variables en Stata & Descripción \\
\hline$Y_{i}$ & Analfabeta & $\begin{array}{l}\text { Dummy, toma el valor de } 1 \text { si el } \\
\text { joven es analfabeto y } 0 \text { en caso } \\
\text { contrario. }\end{array}$ \\
\hline$D_{i}$ & $\begin{array}{l}\text { noedadescolar; yatermino; faltadedine- } \\
\text { ro; oficioshogar; embarazo; inseguridad; } \\
\text { faltacupos; estableciretirado; debtraba- } \\
\text { jar; nolegusta; enfermedad; eduespecial; } \\
\text { habandolrh; duhombre; vivesinpadre; } \\
\text { viveconmadre; vivesinmadre; pprimaria; } \\
\text { psecundaria; edad2. }\end{array}$ & $\begin{array}{l}\text { Variables explicativas, las cua- } \\
\text { les toman el valor de uno (1) } \\
\text { si cumplen con la descripción } \\
\text { de la variable y cero }(0) \text { en caso } \\
\text { contrario. }\end{array}$ \\
\hline$W_{i}$ & edad & $\begin{array}{l}\text { Variable ordinal, así: } 1 \text { sí }>=14 \& \\
<18 ; 2 \text { sí }>=18 \&<24 ; 3 \text { sí }>=24 \\
\&<=28 .\end{array}$ \\
\hline$\mu_{i}$ & Término de error & $\begin{array}{l}\text { Recoge los factores que afectan } \\
\text { a la variable dependiente y que } \\
\text { han sido excluidos del modelo. }\end{array}$ \\
\hline
\end{tabular}

Fuente: el autor

Según Wooldridge (2010: 576) En el modelo probit, $G$ es la función de distribución acumulada (Fda) normal estándar, que se expresa como una integral:

$$
\boldsymbol{G}(\mathbf{z})=\Phi(\mathbf{z}) \equiv \int_{-\infty}^{\mathbf{z}} \emptyset(\mathbf{v}) \mathbf{d} \mathbf{v}
$$


Donde $\oint(z)$ es la densidad normal estándar

$$
\Phi(z)=(2 \pi)^{-1 / 2} \exp \left(-z^{2} / 2\right)
$$

$G$ asegura que (b) esté estrictamente entre cero y uno para todos los valores de los parámetros y las $\mathrm{X}_{\mathbf{k}}$.

$P$ (analfabeto $\left._{i}=1 \mid D_{i}, W_{i}\right)=G\left(\beta_{1}+\beta_{1}\right.$ noedadescolar $_{i}+\beta_{1}$ yatermino $_{i}$ $+\beta_{1}$ faltadedinero $_{i}+\beta_{1}$ oficioshogar $_{i}+\beta_{1}$ embarazo $_{i}+\beta_{1}$ inseguridad $_{i}$ $+\beta_{1}$ faltacupos $_{i}+\beta_{1}$ estableciretirado $_{i}+\beta_{1}$ debtrabajar $_{i}+\beta_{1}$ nolegusta $_{i}$ $+\beta_{1}$ enfermedad $_{i}+\beta_{1}$ eduespecial ${ }_{i}+\beta_{1}$ habandolrh $_{i}+\beta_{1}$ duhombre $i++$ $\beta_{1}$ vivesinpadre $i+\beta_{1}$ viveconmadre $i+\beta_{1}$ vivesinmadre $i+\beta_{1}$ pprimariai + $\beta_{1}$ psecundariai $+\beta_{1}$ edadi $+\beta_{1}$ edad $\left.2 i+\mu_{i}\right)$

\section{RESULTADOS}

La estimación de los modelos, como ya se ha mencionado, se realiza a través del modelo Probit cuya función de distribución acumulada es normal estándar, la estimación se efectúa mediante el paquete econométrico Stata 12, el cual es ampliamente utilizado por investigadores de distintas disciplinas, especialmente de la Ciencia Económica.

\subsection{VULNERABILIDAD A NO EDUCARSE (ANALFABETISMO)}

La estimación en primera instancia procura explicar los determinantes de la vulnerabilidad a no educarse, para ello se ha tenido en cuenta los indicadores propuestos en la dimensión y en este sentido se ha corrido el primer modelo para definir las causas que determinan el hecho de que un joven sea analfabeta/o. El porcentaje de jóvenes que no saben leer y escribir es de $2,16 \%$, si bien resulta relativamente bajo, es preocupante en la medida en que aún existe un frente que se debe tratar.

La tabla 1 muestra la salida del programa econométrico, los valores estimados se pueden interpretar directamente en términos de probabilidades, puesto que representan los cambios marginales en la dummy dependiente cuya distribución es conocida $(0$ - 1). Con el fin de mejorar la estimación, se ha corrido el modelo con errores estándar robustos, los cuales corrigen la matriz de varianzas y covarianzas y a la postre evitan problemas heterocedásticos.

Cabe anotar que el número de variables están inmersas en ciertas categorías, v. gr., una de las primeras categorías que se consideró, corresponde a las causas por las cuales los jóvenes son analfabetos. Atendiendo al marco teórico de los modelos con regresada binaria en función de variables cualitativas que sugiere incorporar al modelo cuantas variables dicótomas haya en una categoría (menos una), "la categoría a la cual no se asigna variable dicótoma se conoce como categoría base o

5. Si no se respeta esta regla se provocará lo que se conoce como la trampa de la variable dicótoma; es decir, se tendrá una situación de perfecta colinealidad o perfecta Multicolinealidad, si hay más de una relación exacta entre las variables. (Gujarati y Porter, 2010: 281). 
de referencia” (Gujarati y Porter, 2010: 281), por tanto, las interpretaciones, siempre se harán en términos de la variable excluida céteris páribus.

Tabla 1. Modelo Probit para determinar la vulnerabilidad de los jóvenes a no educarse por analfabetismo

\begin{tabular}{|c|c|c|c|c|}
\hline$P\left(\right.$ analfabeto $\left._{i}=1 \mid D_{i}, W_{i}\right)$ & $\mathrm{dF} / \mathrm{dx}$ & $\begin{array}{l}\text { Robust } \\
\text { Std. Err. }\end{array}$ & $\mathbf{z}$ & $P>z$ \\
\hline no está en edad escolar* & 0,0383768 & 0,0217091 & 2,84 & 0,004 \\
\hline ya terminó* & $-0,0173058$ & 0,0020198 & $-3,56$ & 0,000 \\
\hline falta de tiempo* & $-0,0170587$ & 0,0038978 & $-3,57$ & 0,000 \\
\hline oficios del hogar* & $-0,0033109$ & 0,0054978 & $-0,56$ & 0,576 \\
\hline estado de embarazo* & $-0,0113914$ & 0,0032445 & $-1,83$ & 0,068 \\
\hline inseguridad en el establecimiento edu.* & 0,0165185 & 0,0282264 & 0,80 & 0,425 \\
\hline falta de cupos* & $-0,0071802$ & 0,0063174 & $-0,82$ & 0,41 \\
\hline no existe centro educativo cercano* & 0,0021441 & 0,0111825 & 0,20 & 0,839 \\
\hline debe trabajar* & $-0,0121997$ & 0,0042603 & $-2,46$ & 0,014 \\
\hline no le gusta o desinterés por el estudio* & 0,0183188 & 0,0111765 & 2,20 & 0,028 \\
\hline por enfermedad* & 0,1711626 & 0,0596656 & 5,84 & 0,000 \\
\hline necesita educación especial* & 0,5058646 & 0,0905748 & 9,89 & 0,000 \\
\hline abandono del lugar de residencia* & $-0,0007958$ & 0,0119777 & $-0,06$ & 0,948 \\
\hline dummy hombre* & 0,0086157 & 0,0028904 & 3,21 & 0,001 \\
\hline vive sin el padre* & 0,0013352 & 0,0026519 & 0,49 & 0,623 \\
\hline vive con la madre* & $-0,0060908$ & 0,0046243 & $-1,24$ & 0,216 \\
\hline vive sin la madre* & $-0,0047464$ & 0,0056711 & $-0,89$ & 0,376 \\
\hline edu. Primaria del padre* & $-0,0196119$ & 0,0028126 & $-8,07$ & 0,000 \\
\hline edu. Secundaria del padre* & $-0,0178537$ & 0,0021570 & $-6,43$ & 0,000 \\
\hline edad & 0,0083831 & 0,0103866 & 0,81 & 0,419 \\
\hline edad2 & $-0,0017379$ & 0,0024409 & $-0,71$ & 0,476 \\
\hline Log pseudolikelihood & $-852,36021$ & & & \\
\hline Number of obs & 7730 & & & \\
\hline Wald chi2 (21) & 481,09 & & & \\
\hline Prob > chi2 & 0,0000 & & & \\
\hline Pseudo R2 & 0,2573 & & & \\
\hline
\end{tabular}

$\left({ }^{*}\right) \mathrm{dF} / \mathrm{dx}$ is for discrete change of dummy variable from 0 to 1

$\mathrm{z}$ and $\mathrm{P}>|\mathrm{z}|$ correspond to the test of the underlying coefficient being 0

Fuente: el autor, con datos de la GEIH y ENCV, DANE. 
Según el estadístico de Wald, las variables explicativas tomadas en su conjunto son explicativas del evento del analfabetismo; por su parte, el Pseudo R2 indica que las variaciones en la probabilidad de que éste suceda (variable regresada), está siendo explicada en un $25 \%$ por las variaciones de las variables regresoras.

Una revisión a la significancia individual de las variables, indica, en cuanto a las posibles causas que explican el analfabetismo en los jóvenes en Colombia, que las variables: no está en edad escolar, ya terminó, falta de tiempo, estado de embarazo, debe trabajar, no le gusta, por enfermedad, requiere educación especial, variable dummy hombre y la educación primaria y secundaria del padre, resultaron ser altamente significativas en el modelo ${ }^{6}$.

Es interesante observar como aquellos jóvenes que consideran no estar en edad escolar, aumentan la probabilidad de ser analfabetos en el 3,8\%; por su parte, considerar que ya terminó o falta de tiempo, disminuyen la probabilidad de ser analfabeta, al igual que estar en embarazo. Tener un trabajo, disminuye la probabilidad de ser analfabeto, esta situación se asocia al hecho mismo de que para insertarse al mercado laboral, por lo menos, se requiere saber leer y escribir.

De otro lado, variables como: no le gusta, enfermedad o requerir una educación especial, aumentan la probabilidad de ser analfabeta/o a no saber leer y escribir. Respecto a la variable género, es posible señalar que los hombres aumentan la probabilidad de ser analfabetos en el 0,8\% respecto a las mujeres. En cuanto a la educación del padre del joven, se puede observar que tiene una incidencia bastante importante, puesto que si el padre cuenta con educación primaria completa o incompleta aumenta la probabilidad en el 1,9\% de que su hijo sea analfabeto; por su parte, si el padre cuenta con educación secundaria completa o incompleta aumenta la probabilidad de que su hijo sea analfabeto en el 1,7\%, no obstante, es una proporción relativamente menor si se la compara con un padre que sólo cuenta con educación primaria.

Si bien esta primera categoría explica en mayor medida el evento de que un joven no estudie, como se verá más adelante, para el caso del analfabetismo, se la ha tenido en cuenta con el ánimo de vislumbrar posibles razones que llevan a que un joven no sepa leer y escribir. En este punto cabe resaltar que el porcentaje de jóvenes en Colombia que sabe leer y escribir es del 97,84\% en comparación con el $2,16 \%$ que no sabe (ver Tabla 2) y en consecuencia se considera analfabeta/o.

Tabla 2. Proporción de jóvenes en Colombia entre 14 y 28 años que no sabe leer y escribir, año 2012

\begin{tabular}{|c|r|r|r|}
\hline ¿Sabe leer y escribir? & \multicolumn{1}{c|}{ Freq. } & Per cent & Cum. \\
\hline Sí & 18,371 & 97,84 & 97,84 \\
\hline No & 0,406 & 2,16 & 100,00 \\
\hline Total & 18,777 & 100,00 & \\
\hline
\end{tabular}

Fuente: Cálculo del autor, con datos de la GEIH y ENCV, DANE.

6. Las variables que no se mencionen será porque desde el punto de vista estadístico resultan ser insignificantes. 
La situación expuesta es bastante lógica desde el punto de vista de la vulnerabilidad a no educarse y en consecuencia a perpetuarse en la pobreza, dado que desafortunadamente existen factores que de por si son negativos, asociados sobre todo a su medio externo, y que a la postre influyen de manera directa en la condición de analfabetismo del 2,16\% de los jóvenes.

\subsection{VULNERABILIDAD A NO EDUCARSE (INASISTENCIA ESCOLAR)}

Resulta interesante observar como: no estudiar, presenta un sin número de razones, variables que ya fueron utilizadas como explicación de la vulnerabilidad por analfabetismo. Para el indicador de inasistencia escolar, para efectos de análisis se lo ha planteado en términos negativos, esto dado que deja ver una relación más clara a la hora de evaluar la vulnerabilidad a no educarse.

Entre las causas por las cuales los jóvenes entre 14 y 28 años no asisten a un establecimiento educativo es principalmente porque: les falta dinero o los costos educativos son muy elevados, deben encargarse de los oficios del hogar, necesitan trabajar y porque no les gusta o no les interesa el estudio (ver Tabla 3).

Tabla 3. Principales razones para que no estudie un joven entre los 14 y 28 años, año 2012

\begin{tabular}{|l|r|r|r|}
\hline \multicolumn{1}{|c|}{ Principal razón para que no estudie } & \multicolumn{1}{c|}{ Freq. } & Percent & \multicolumn{1}{c|}{ Cum. } \\
\hline considera que no está en edad escolar & 177 & 1,59 & 1,59 \\
\hline considera que ya terminó & 1029 & 9,23 & 10,82 \\
\hline falta de dinero o costos educativos elevados & 2916 & 26,15 & 36,97 \\
\hline debe encargarse de los oficios del hogar & 1452 & 13,02 & 49,99 \\
\hline por embarazo & 329 & 2,95 & 52,94 \\
\hline inseguridad o malos tratos en el establecimiento educ. & 50 & 0,45 & 53,39 \\
\hline falta de cupos & 167 & 1,50 & 54,89 \\
\hline no existe centro educativo cercano & 151 & 1,35 & 56,24 \\
\hline necesita trabajar & 2775 & 24,88 & 81,12 \\
\hline no le gusta o no le interesa el estudio & 1384 & 12,41 & 93,53 \\
\hline por enfermedad & 133 & 1,19 & 94,72 \\
\hline necesita educación especial & 120 & 1,08 & 95,80 \\
\hline tuvieron que abandonar el lugar de residencia & 64 & 0,57 & 96,37 \\
\hline otra razón. ¿Cuál? & 406 & 3,64 & 100,0 \\
\hline Total & 1153 & 100,0 & \\
\hline
\end{tabular}

Fuente: Cálculo del autor, con datos de la GEIH y ENCV, DANE. 
Resulta preocupante observar como a un nivel descriptivo, las variables: falta de dinero y/o los costos elevados de la educación sumado a la necesidad de trabajar resultan ser las principales causas que explican la no asistencia a un establecimiento educativo, situación que redunda en el deterioro de la calidad de vida de ser pobre y al mismo tiempo de perpetuarse en la pobreza, lo que al final terminarían replicando la historia de sus padres que a propósito ya se comprobó que el solo hecho de contar con primaria o secundaria inciden de manera importante en la vulnerabilidad a no educarse.

Un tratamiento econométrico del indicador de inasistencia escolar en la medición de la vulnerabilidad a no educarse, contribuye a dar luces sobre los factores que explican el hecho de que el 59,4\% no asista a una escuela, colegio o universidad. Para efectos de análisis y con el fin de comprender de mejor manera el fenómeno, además de algunas variables explicativas contempladas en el modelo de analfabetismo, para el modelo de la inasistencia escolar, se ha incorporado la principal actividad en la cual emplean el tiempo los jóvenes. Así, se puede observar que los jóvenes ocupan la mayor parte del tiempo en la semana bajo análisis, trabajando con el 36\%; estudiando 35\% y en los oficios del hogar el 20\%, (ver Tabla 4).

Tabla 4. Principal actividad en la que un joven ocupó la mayor parte del tiempo la semana de referencia, año 2012

\begin{tabular}{|l|r|r|r|}
\hline $\begin{array}{c}\text { Actividad en que ocupó la mayor parte del } \\
\text { tiempo la semana de referencia }\end{array}$ & Freq. & Percent & Cum. \\
\hline trabajando & 6812 & 36,28 & 36,28 \\
\hline buscando trabajo & 807 & 4,30 & 40,58 \\
\hline estudiando & 6577 & 35,03 & 75,61 \\
\hline oficios del hogar & 3879 & 20,66 & 96,27 \\
\hline incapacitado permanente para trabajar & 140 & 0,75 & 97,02 \\
\hline otra actividad. ¿Cuál? & 562 & 2,99 & 100,0 \\
\hline Total & 18777 & 100,0 & \\
\hline
\end{tabular}

Fuente: Calculo del autor, con datos de la GEIH y ENCV, DANE.

Por su parte, siguiendo con el análisis de las variables que explican la vulnerabilidad a no educarse por inasistencia escolar se realiza la especificación de un segundo modelo (ver Cuadro 4); el planteamiento formal y la metodología empleada se deriva de la utilizada en el primer modelo.

Donde la probabilidad de no estudiar o las dificultades que existen para acceder a educación dependen de un conjunto de variables dicótomas, ordinales y cuantitativas, la cuales contribuyen a comprender el fenómeno de la vulnerabilidad a no educarse por inasistencia (ver Ecuación $\boldsymbol{c}$ ). 
Cuadro 4. Especificación de variables del modelo de Vulnerabilidad a no educarse por inasistencia escolar

\begin{tabular}{|c|l|l|}
\hline $\begin{array}{c}\text { Variable en } \\
\text { el modelo }\end{array}$ & \multicolumn{1}{|c|}{ Variables en Stata } & \multicolumn{1}{|c|}{ Descripción } \\
\hline $\boldsymbol{Y}_{\boldsymbol{i}}$ & noestudia & $\begin{array}{l}\text { Dummy, toma el valor de 1 si el } \\
\text { joven no estudia y 0 en caso con- } \\
\text { trario. }\end{array}$ \\
\hline $\boldsymbol{D}_{\boldsymbol{i}}$ & $\begin{array}{l}\text { duhombre; vivesinpadre; viveconmadre; } \\
\text { vivesinmadre; pprimaria; psecundaria; } \\
\text { ptec_o_tecg; trabajando; desempleado; } \\
\text { inactivo; edad; edad2. }\end{array}$ & $\begin{array}{l}\text { Variables explicativas, las cuales } \\
\text { toman el valor de uno (1) si cum- } \\
\text { plen con la descripción de la va- } \\
\text { riable y cero (0) en caso contrario. }\end{array}$ \\
\hline $\boldsymbol{W}_{\boldsymbol{i}}$ & edad & $\begin{array}{l}\text { Variable ordinal, así: 1 sí }>=14 \text { \& } \\
<18 ; 2 \text { sí >=18 \& <24; 3 sí >=24 \& } \\
<=28 .\end{array}$ \\
\hline$\mu_{i}$ & Término de error & $\begin{array}{l}\text { Recoge los factores que afectan } \\
\text { a lariable dependiente y que } \\
\text { han sido excluidos del modelo. }\end{array}$ \\
\hline
\end{tabular}

Fuente: el autor

$P\left(\right.$ noestudia $\left._{i}=1 \mid D_{i}, W_{i}\right)=G\left(\beta 1+\beta 1\right.$ duhombre $_{i}+\beta$ viveconpadre $_{i}+$ $\beta_{1}$ vivesinpadre $_{i}+\beta$ 1viveconmadre $_{i}+\beta$ 1 vivesinmadre $_{i}+\beta_{1}$ pprimaria $_{i}$ $+\beta 1$ psecundaria $_{i}+\beta 1$ ptéc_o_tecg $_{i}+\beta$ trabajando $_{i}+\beta$ 1desempleado $_{i}$ $+\beta$ inactivo $_{i}+\beta$ Bedad $_{i}+\beta$ edad $\left._{i}+\mu_{i}\right)$

En general el modelo presenta un ajuste bastante bueno, a juzgar por el estadístico Wald y del pseudo R2, la significancia estadística individual es del 95\% e incluso 99\% en las siguientes variables: dummy de hombre, vive sin padre, vive con madre, padre con educación secundaria, padre con educación técnica o tecnológica, trabajando, desempleado, inactivo, edad y edad2 (ver Tabla 5).

El análisis en términos de probabilidades indica que ser hombre joven reduce la probabilidad de no estudiar por inasistencia a un establecimiento educativo en el $10,4 \%$ respecto a las mujeres jóvenes, lo que significa que aún las mujeres presentan algunas dificultades para asistir a un establecimiento educativo, esto a pesar de los importantes avances que se han venido realizando en términos de inclusión, democracia, garantía de derechos y equidad de género.

Por su parte, los jóvenes que viven sin su padre reducen la probabilidad a no estudiar en el $0.3 \%$, mientras que si viven con la madre dicha probabilidad se reduce en un $17 \%$, esto se debe tal vez al hecho mismo de que los jóvenes que cuentan con un hogar en donde la madre hace las veces de madre cabeza de familia o simplemente viven con ella, la probabilidad de que no estudien es mucho más baja en comparación con otros estadios, esto se explica porque la madre ocupa un papel fundamental en el desarrollo personal de sus hijos. 
Tabla 5. Modelo probit para determinar la vulnerabilidad de los jóvenes a no educarse por inasistencia escolar

\begin{tabular}{|c|c|c|c|c|}
\hline$P\left(\right.$ noestudia $\left._{i}=1 \mid D_{i}, W_{i}\right)$ & $\mathrm{dF} / \mathrm{dx}$ & $\begin{array}{c}\text { Robust Std. } \\
\text { Err. }\end{array}$ & $\mathbf{z}$ & $P>z$ \\
\hline dummy hombre* & $-0,1043023$ & 0,0118316 & $-8,73$ & 0,000 \\
\hline vive sin el padre* & $-0,0325090$ & 0,0125071 & $-2,54$ & 0,011 \\
\hline vive con la madre* & $-0,1703131$ & 0,0285152 & $-6,04$ & 0,000 \\
\hline vive sin la madre* & 0,0118130 & 0,0275958 & 0,43 & 0,668 \\
\hline edu. Primaria del padre* & $-0,0057378$ & 0,0112083 & $-0,51$ & 0,608 \\
\hline edu. Secundaria del padre* & $-0,1292245$ & 0,0143388 & $-9,40$ & 0,000 \\
\hline edu. Técnica o tecnológica del padre* & $-0,3209201$ & 0,0416539 & $-7,88$ & 0,000 \\
\hline trabajando* & $-0,2268876$ & 0,0402753 & $-5,66$ & 0,000 \\
\hline desempleado* & $-0,0262365$ & 0,0546820 & $-0,49$ & 0,624 \\
\hline inactivo* & $-0,5137405$ & 0,0291621 & $-14,6$ & 0,000 \\
\hline edad & 0,5745899 & 0,0416765 & 14,03 & 0,000 \\
\hline edad2 & $-0,098030$ & 0,0104140 & $-9,52$ & 0,000 \\
\hline Log pseudolikelihood & $-4811,8953$ & & & \\
\hline Number of obs & 11562 & & & \\
\hline Wald chi2 (21) & 3190,14 & & & \\
\hline Prob > chi2 & 0,0000 & & & \\
\hline Pseudo R2 & 0,3392 & & & \\
\hline
\end{tabular}

$\left({ }^{*}\right) \mathrm{dF} / \mathrm{dx}$ is for discrete change of dummy variable from 0 to 1

$\mathrm{z}$ and $\mathrm{P}>|\mathrm{z}|$ correspond to the test of the underlying coefficient being 0

Fuente: Calculo del autor, con datos de la GEIH y ENCV, DANE.

La categoría educación del padre como variable explicativa de la probabilidad de que un joven no estudie, resulta de gran interés, dado que en la medida en que el padre incrementa su nivel educativo, la probabilidad de que sus hijos NO estudien se reduce cada vez más, situación bastante positiva, pues lo que se espera es que un hogar compuesto por padres educados perciban mejores ingresos. De manera que, las razones que se identificaban como explicativas de la no asistencia a un establecimiento educativo como la falta de dinero o tener que trabajar, ante un escenario con padres educados, se reducen; además, a mayor educación, se valora aún más este activo y en consecuencia, se estimula a los hijos desde pequeños en ambientes de lectura y aprendizaje.

Por su parte, tener trabajo reduce la probabilidad de no estudiar en el 22,6\%, un porcentaje bastante alto, pues como ya se han mencionado, contar con un empleo, amplía las posibilidades para prepararse y educarse aprovechando horarios, 
ya sea nocturnos o de fines de semana. La variable inactivos resulta ser una de la más representativas porque se debe recordar que precisamente en dicha variable caben situaciones en la que los jóvenes son: estudiantes, se dedican a oficios del hogar, son rentistas, hijos de jubilados, o tienen alguna discapacidad para trabajar. Así, cuando se evalúa la actividad en la que un joven ocupó la mayor parte del tiempo en la semana de referencia, la variable estudiando (35.03\%), hace parte de la categoría de inactivos, por tanto, es de esperarse que estar en dicha condición, disminuya la probabilidad a no estudiar en el 51\%.

Finalmente, la edad indica que a medida que el joven adquiere más años, aumenta la probabilidad de no estudiar en el 57\%, esta característica coincide con lo señalado por los jóvenes cuando les indagaron las razones del porqué no estudiaban, a lo cual respondieron que no estaban en la edad de estudio. Además, muchos jóvenes que deberían estar en un establecimiento de educación superior y no están, se llenan cada vez más de prejuicios para ingresar a un establecimiento con edades que superan el promedio de edad de los estudiantes de instituciones educativas.

\section{CONCLUSIONES}

Las políticas públicas encaminadas a reducir las brechas de desigualdad, pobreza y dirigidas a prevenir escenarios negativos, de riesgo, de shocks y amenazas desde la dimensión de la educación, deberán centrar los esfuerzos sobre los factores fundamentales que explican la inasistencia escolar, a saber: la falta de dinero y/o los costos educativos elevados y porque necesita trabajar, en cualquier caso, garantizar la gratuidad en la educación pública en Colombia debería ser una política de estado si se quiere mejorar la educación; una educación de calidad, sin duda contribuye al desarrollo de un país. En este frente, se destaca el programa Ser Pilo Paga anunciado por el Gobierno Nacional ${ }^{7}$ en donde los estudiantes con puntajes

7. En cuanto a políticas relacionadas con el acceso al sistema educativo, terminando el presente estudio, el Gobierno Nacional en cabeza del Ministerio de Educación realizó un anuncio el 21 de mayo de 2015 denominado “Tú Eliges”, el programa es una nueva línea de crédito que cubre la totalidad de la matrícula y ofrece cinco nuevas modalidades de pago; además, la falta de codeudor ya no sería un problema, pues quienes no lo tengan, mediante el pago adicional del $2 \%$ del total de la matrícula se podría acceder a un fondo de garantías para cubrirlo, el programa se enfoca principalmente en estratos 1,2 y 3 cuyos jóvenes demuestren tener un puntaje igual o superior a 280 en las Pruebas Saber 11. No obstante, si bien se ha venido realizando importantes avances que abarcan diferentes frentes en la atención que merecen los jóvenes, las políticas, si se quiere mejorar las condiciones de vida de los y las jóvenes se deben diseñarse contemplando problemas estructurales que los atañen para dejar de tomar decisiones basándose en los efectos y empezar a tratar las causas del problema. (Min. Educación, 2015). De otra parte, Las estrategias que ha implementado el Gobierno Nacional para mejorar y revertir la tendencia en los sectores de capacitación y educación superior, según el CONPES Social 100, en materia de capacitación, el SENA ha concentrado su atención en el diseño e implementación de programas para la inserción y la reinserción laboral para poblaciones vulnerables como: programa Jóvenes en Acción y Jóvenes Rurales, entre otros. En el caso de la educación superior se menciona los proyectos de Centros Regionales de Educación Superior (CERES) que buscan descentralizar la oferta de este nivel educativo y llegar a las regiones que concentra la población actualmente excluida del servicio de educación superior e igualmente a través del Programa de Crédito Educativo (ACCES), se pretendía atender a una mayor proporción de jóvenes de bajos ingresos. (Conpes Social 100, 2006: 7). 
mayores a 310 en las pruebas del 3 de agosto de 2014 y pertenecientes al Sisbén según lo establecido por el Icetex, podrán acceder a una beca para programas de educación superior en instituciones acreditadas de alta calidad.

De otra parte, la generación de empleo digno para la población económicamente activa (PEA) joven, contribuye a reducir las cargas de dependencia en el hogar, aliviando gastos y al mismo tiempo motivando en los jóvenes la importancia de estudiar y de prepararse constantemente para mejorar su situación económica y superar la pobreza. En consecuencia, la opción de trabajar, dejaría de ser una necesidad y pasaría a ser una decisión, dado que la generación de más y mejor empleo, distribuiría las cargas de responsabilidad entre los miembros del hogar.

En cuanto a la vulnerabilidad por inasistencia escolar, se logró evidenciar que los hombres tienen una menor probabilidad a no estudiar que las mujeres, de manera que se deben diseñar políticas más incluyentes que le apunten a reducir las brechas de género. Por su parte, la madre también se convierte en un elemento fundamental de la familia debido a que su presencia reduce la probabilidad de los jóvenes a no estudiar, también se corroboró situaciones como la educación del padre, la cual influye positivamente en la probabilidad de que sus hijos estudien, pues se logró observar que a mayor educación del padre se reduce la probabilidad de no estudiar.

El trabajo, entendido como un medio de sustento, también resultó importante entre los jóvenes, puesto que dicha condición reduce ampliamente las probabilidades de no estudiar, ser inactivo, dada su naturaleza también la disminuye. La edad, presentó una relación positiva con la probabilidad de no estudiar, lo que significa que tener mayor edad aumenta el riesgo de no asistir a un establecimiento educativo en el $57 \%$, porcentaje bastante alto teniendo en cuenta que el rango de edad es de 14 a 28, por lo que las políticas sobre educación, deben considerar incentivos para que el mayor de edad se incorpore al sistema educativo.

Finalmente, dada la escasez de estudios respecto al tema de la vulnerabilidad en Colombia y con el propósito de continuar consolidando un marco teórico sólido, se hace necesario seguir avanzando en los interrogantes y los desafíos que propone la medición de la vulnerabilidad, todo con el fin de contribuir al diseño de políticas públicas pertinentes y efectivas. 


\section{REFERENCIAS}

1. BUSSO, G. (2001). Vulnerabilidad Social: Nociones e implicancias para Latinoamérica a inicios del siglo $X X I$. Seminario internacional las diferentes expresiones de la vulnerabilidad social en América Latina y el Caribe. Santiago de Chile: CEPAL.

2. CASTAÑO, Lina (2007). Una aproximación a la vulnerabilidad, Departamento Nacional de Planeación, Dirección de Desarrollo Social Grupo de Calidad de Vida. República de Colombia, Bogotá D.C., Impresión Gráficas Ducal Ltda.

3. COLOMBIA (2013). Ley estatutaria 1622 de 2013. Estatuto de Ciudadanía Juvenil.

4. COLOMBIA (2015). Ministerio de Trabajo, Gobierno patrocinará primer empleo a 40 mil jóvenes entre 18 y 28 años, Comunicado del 9 de febrero de 2015. Disponible En: http://www.mintrabajo.gov.co/ febrero-2014/4187-gobierno-patrocinara-primer-empleo-a-40-mil-jovenes-entre-18-y-28-anos.html, recuperado el 27 de febrero de 2015.

5. COMISIÓN ECONÓMICA PARA AMÉRICA LATINA Y EL CARIBE, CEPAL (1999). Marco Conceptual sobre activos, Vulnerabilidad y Estructura de Oportunidades.

6. DEPARTAMENTO NACIONAL DE PLANEACIÓN (2006). Conpes Social 100. Bogotá, D.C.: DNP.

7. Documento preparado por la Oficina de CEPAL en Montevideo, con el apoyo financiero del PNUD, en el marco del Proyecto URU/97/017. Apoyo a la implementación del Programa de Acción de la Cumbre Mundial sobre Desarrollo Social..

8. ESTUPIÑÁN, V. y GUERRERO, Gissela (2013). Medición de la Vulnerabilidad de los Hogares en Colombia: Una Propuesta Metodológica. Aplicación al Municipio de Pasto, Año 2012. San Juan de Pasto, Colombia: Universidad de Nariño. Recuperado el 25 de mayo de 2015.

9. FIGUEIRA, C. (2001). Estructura de Oportunidades y Vulnerabilidad Social Aproximaciones Conceptuales Recientes. Seminario internacional, las diferentes expresiones de la vulnerabilidad social en América Latina y el Caribe. Santiago de Chile: Naciones Unidas.

10. GOBIERNO DE CHILE, MIDEPLAN (2003). Encuesta de Caracterización Socioeconómica Nacional, Manual de Stata.

11. GUJARATI, Damodar y PORTER, Dawn (2010). Econometría. 5 ed. México D.F: McGraw-Hill Interamericana. $921 \mathrm{p}$.

12. JEUDE, V. L. (2005). Análisis y estrategias para poblaciones rurales con mayor vulnerabilidad socioeconómica en Centroamérica. Serie de Publicaciones Ruta. Centroamérica: Infoterra Editores S.A.

13. LAS NOTICIAS DE STATA (2011). Revista enero, febrero, marzo de 2011, Vol. 26 No. 1.

14. NIEVES, M. (2011). El desafío de un sistema nacional de cuidados para el Uruguay. Serie Seminarios y Conferencias (66). Santiago de Chile: Naciones Unidas.

15. NOVA, D., BERMÚDEZ, R., \& GORDILLO, C.Y. (s.f.). Algoritmo para la conformación del ingreso per cápita para la medición de pobreza a partir de la encuesta continua de hogares - ECH (2002-2005) y de la gran 
encuesta integrada de hogares - GEIH (2008-2010). Misión para el Empalme de la Series de Empleo Pobreza y Desigualdad - MESEP, con base en la metodología definida por esta misión. DANE.

16. OIT. (2015). Formalizando Ia Informalidad Juvenil. Experiencias Innovadoras en América Latina y el Caribe. Lima: Oficina de la OIT para América Latina y el Caribe.

17. PAGANO, Juan; ROSSI, Lanina; ROSSI, Máximo y TRIUNFO, Patricia (s.f.). Introducción a Stata, notas docentes, nota docente No. 17. Universidad de la República, Facultad de Ciencias Sociales, Departamento de Economía.

18. PIZARRO, R. (2001). La vulnerabilidad social y sus desafíos: una mirada desde América Latina. Serie estudios estadísticos y prospectivos. Santiago de Chile, Chile: Naciones Unidas.

19. RODRIGUEZ, Carolina; CÁCERES, José, (2007). Modelos de elección discreta yespecificaciones ordenadas: una reflexión metodológica, Estadística Española, Vol. 49, Núm. 166, 2007, pp. 451 a 471.

20. ROJA, F. I. (2007). Análisis de Vulnerabilidad y Capacidad, Lecciones aprendidas y recomendaciones. Agenda Global de la Federación Internacional (2006-2010). Ginebra, Suiza.

21. ROJO, José (2008). Curso de Introducción al paquete Stata (versión 9.1 SE), Unidad de análisis estadístico, Centro de Ciencias Humanas y Sociales, Consejo Superior de Investigaciones Científicas (CCHS, CSIC), Madrid.

22. ROSALES, et. al. (2013). Fundamentos de econometría intermedia:teoría y aplicaciones. Bogotá: Ediciones Uniandes, 420p.

23. SOJO, Ana (2012). Desafío para la medición de la vulnerabilidad y las políticas públicas pertinentes, Vol. 3 Núm. 2.

24. TABORDA, R. (2011). Estimaciones y Comandos Stata. Universidad del Rosario, Facultad de Economía.

25. VÁSQUEZ, Javiera (s.f.). Curso de nivelación Stata Magister en políticas públicas. Centro de Micro datos. Departamento de Economía, Universidad de Chile.

26. WOOLDRIDGE, J. (2010). Introducción a la Econometría un Enfoque Moderno (Cuarta ed.). México, D.F.: Cengage Learning Editores, S.A. de C.V.

\section{NETGRAFÍA}

27. BANCO MUNDIAL [En línea]: www.bancomundial.org/

28. MINISTERIO DE EDUCACIÓN [En línea]: http://www.mineducacion.gov.co/

29. MINISTERIO DE TRABAJO [En línea]: http://www.mintrabajo.gov.co/

30. TEMAS MUNDIALES [En línea]: http://www.un.org/es/globalissues/youth/ 